Revue d'histoire de l'Amérique française

REYUE D.HISTOIRE DE L'AMÉRIQUE FRANÇAISE

\title{
DUMONT, Fernand, Jean HAMELIN et Jean-Paul MONTMINY, éd., Idéologies au Canada français 1940-1976. Québec, Les Presses de l’Université Laval, 1981. Trois tomes : 45,00 \$.
}

\section{Jacques Rouillard}

Volume 37, numéro 3, décembre 1983

URI : https://id.erudit.org/iderudit/304187ar

DOI : https://doi.org/10.7202/304187ar

Aller au sommaire du numéro

Éditeur(s)

Institut d'histoire de l'Amérique française

ISSN

0035-2357 (imprimé)

1492-1383 (numérique)

Découvrir la revue

Citer ce compte rendu

Rouillard, J. (1983). Compte rendu de [DUMONT, Fernand, Jean HAMELIN et Jean-Paul MONTMINY, éd., Idéologies au Canada français 1940-1976. Québec, Les Presses de l'Université Laval, 1981. Trois tomes : 45,00 \$.] Revue d'histoire de l'Amérique française, 37(3), 461-463. https://doi.org/10.7202/304187ar d'utilisation que vous pouvez consulter en ligne.

https://apropos.erudit.org/fr/usagers/politique-dutilisation/ 
DUMONT, Fernand, Jean HAMELIN et Jean-Paul MONTMINY, éd., Idéologies au Canada français 1940-1976. Québec, Les Presses de l'Université Laval, 1981. Trois tomes: 45,00\$

Avec ces trois tomes de plus de 1100 pages au total, la boucle est maintenant complétée; les auteurs ont porté à terme leur ambitieux projet commencé en 1971 de tracer une histoire des idéologies au Canada français de 1850 à nos jours. C'est quatre volumes qu'ils nous ont livrés au cours des dix dernières années, apportant ainsi une contribution capitale à l'histoire des idées au Québec. Il est bon de préciser que ce courant historiographique dont les éditeurs sont largement responsables de l'ampleur qu'il a pris au Québec n'a pas d'équivalent au Canada anglais.

Comme pour les volumes antérieurs, certains des textes recueillis dans ces trois derniers tomes proviennent de travaux d'étudiants gradués en histoire et en sociologie, auxquels s'ajoutent les réflexions de Fernand Dumont sur la mutation des idéologies et un tableau de Jean Hamelin et Jean-Paul Montminy traçant à grands traits l'évolution de la situation socio-économique du Québec. Toutefois, les auteurs ont davantage fait appel ici à des collaborateurs extérieurs, la plupart professeurs dans des universités québécoises, ce qui enrichit considérablement ce dernier volume. Ces collaborations, une trentaine, ajoutent de la profondeur aux articles et ont permis un éventail plus vaste de sujets d'analyse. Mais n'ayant pas été soumis à un cadre précis d'analyse, leur approche à l'étude des idéologies varie, ce qui parfois enrichit le volume alors qu'à d'autres occasions, les textes ressemblent davantage à des historiques qu'à des analyses idéologiques. 
Le tome premier aborde la littérature (excellent texte de Maurice Arguin) et la presse à travers quelques journaux dont l'échantillon et les périodes analysées s'avèrent assez épars. Le texte de Pierrette Bouchard-Saint-Amant sur Parti pris est remarquable même si la thèse qu'elle soutient nous apparaît très discutable (elle récuse à la revue son caractère socialiste).

Dans le deuxième tome, les mouvements sociaux sont analysés: revues féminines, professions libérales, coopération agricole, idéologies étudiantes, idéologies du loisir, courant contre-culturel et mouvement syndical. Les articles de Gilles Dussault (professions), Gilles Pronovost (étudiants) et Roger Levasseur (loisir) soulèvent le plus d'intérêt car ils reposent sur un effort de réflexion remarquable. Le texte de Jules Duchastel nous fait découvrir le mouvement contre-culturel des années 70 à travers la revue Mainmise. Peu de recherches ont porté sur ce courant idéologique qui pourtant a eu une énorme influence sur notre société. L'auteur nous laisse malheureusement sur notre appétit accordant beaucoup trop de place à la critique et pas assez à l'analyse du contenu véritable de l'idéologie contre-culturelle. Le tome se termine sur quelques textes de Bernard Solasse consacrés à l'idéologie du mouvement syndical (CSN, FTQ, CEQ, CSD et Fédération des professionnels salariés); ils contiennent des éléments intéressants, mais demeurent largement basés sur des ouvrages secondaires.

Le tome III regroupe les textes sur les partis politiques et l'évolution de l'Église. Le tableau tracé des partis politiques est à peu près complet avec de bons textes sur le Bloc populaire, l'Union nationale, le Parti libéral, le RIN, le Parti québécois et le Parti communiste. On a fait appel à des spécialistes pour rédiger ces articles qui sont une excellente introduction à l'histoire politique de la période. Ils ont réussi à présenter des textes éclairants et concis, sauf celui sur le Parti communiste qui est démesurément long (114 pages). La lecture de l'idéologie du Parti québécois nous fait comprendre jusqu'à quel point l'épreuve du pouvoir peut faire vieillir un parti politique. Sur la situation de l'Église, le texte du Père Richard Arès, davantage un récit qu'une analyse idéologique, déçoit parce qu'il n'apporte pas de réponse à une question centrale: pourquoi la société québécoise s'est-elle décléricalisée aussi rapidement?

Les précédents volumes de cette collection sur les idéologies ont eu une influence marquante sur l'évolution de l'historiographie au Québec. On ne compte plus les thèses dans les années soixante et soixante-dix qui ont porté sur les idéologies. L'engouement a été tel pour l'histoire des idées que beaucoup de jeunes historiens ont délaissé les études basées sur l'événementiel et les sources d'archives. Il y a là un danger pour la compréhension de notre passé dont évidemment ne sont pas responsables les «éditeurs» des volumes. L'idéologie est rarement un reflet de la réalité; il y a souvent un écart considérable entre elle et le discours des définisseurs de société. Avec l'objectif de susciter l'action et de transformer la société, elle caricature ou déforme carrément les faits. On aurait donc tort d'identifier trop intimement l'histoire du Québec à l'histoire de ses idées; elle n'est qu'un aspect de cette histoire, important certes, mais quand même limité pour la compréhension de notre passé.

Il faut voir aussi que certains groupes sociaux écrivent peu, mais n'en ont pas moins une influence prépondérante sur l'évolution de la société. Je pense en particulier à la bourgeoisie d'affaires, dont le poids, depuis la fin du XIX ${ }^{\mathrm{e}}$ 
siècle, est beaucoup plus important chez les francophones qu'on peut le croire. Malheureusement, aucun texte n'est consacré à un de ses organes, pas plus dans ces trois derniers tomes que dans les volumes antérieurs. Par ailleurs, les publications clérico-conservatrices sont si abondamment étudiées que le lecteur n'a pas de mal à croire à l'unanimité idéologique ou au «retard des mentalités» à s'adapter à l'industrialisation.

Dans la même veine, la presse libérale (La Presse, La Patrie, Le Soleil, etc) n'a pas reçu l'attention qu'elle méritait dans les trois premiers volumes. Paule Duchesneau trace au tome III de ce volume un rappel historique de l'idéologie du Parti libéral au début du siècle. On y apprend qu'elle s'inspire du libéralisme et que le progrès économique est «l'élément moteur» de sa pensée. La presse libérale à l'époque reprenait ces thèmes qui n'ont rien à voir avec l'idéologie clérico-conservatrice.

À mon point de vue, le libéralisme est loin d'avoir disparu dans la seconde moitié du XIX ${ }^{e}$ siècle; il apparaît même triomphant avec la prospérité du début du $\mathrm{XX}^{\mathrm{e}}$ siècle et les victoires successives du Parti libéral. Craignant de perdre son emprise sur la population qui s'urbanise de plus en plus, l'Église contreattaque en mettant sur pied les mouvements d'action catholique soutenus par des quotidiens catholiques dans les principales villes de la province. La crise économique de 1929 ébranle, au Québec comme ailleurs, le libéralisme, ce qui permet à l'Église d'y promouvoir un modèle d'organisation socio-politique de son crû, le corporatisme, que reprendra l'Action libérale nationale à la suite du Programme de restauration sociale. L'affirmation de Fernand Dumont à l'effet que les années trente marquent «la première révolution tranquille» me laisse sceptique; elles m'apparaissent au contraire comme un renforcement de la pensée conservatrice, un élargissement de ce courant à la «gouverne politique». En effet, les intellectuels catholiques sont imprégnés pendant ces années par l'anticommunisme, l'agriculturisme et le corporatisme (présent même chez le Bloc populaire). Après la guerre, la jeune génération de clercs et de laics catholiques abandonnera la solution corporatisme discréditée par les régimes fascistes; elle tentera alors de réconcilier le catholicisme avec les idées de liberté et de démocratie. De ce cheminement qui sera suivi par les syndicats catholiques, Le Devoir et les mouvements d'action catholique, on ne trouve malheureusement pas de traces dans les textes retenus pour ce dernier volume.

Il est évidemment facile, assis à son bureau, de reprocher aux instigateurs des volumes d'avoir négligé telle période, tel mouvement ou tel courant idéologique. Ils n'avaient pas d'ailleurs, comme ils le disent en introduction, la prétention de présenter un ouvrage exhaustif. Il leur a fallu composer avec les travaux d'étudiants remis en séminaire et les textes de spécialistes qui ont bien voulu répondre à leur appel. L'unité du volume en souffre, mais ils ne se sont pas si mal tirés d'affaire, compte tenu que les ressources intellectuelles en milieu francophone sont limitées. Leur démarche auprès de spécialistes a permis de réunir plusieurs textes de première valeur qui autrement n'auraient pas vu le jour. Comme nous le faisions remarquer plus tôt, leur entreprise a déjà suscité de nombreuses études sur les idéologies dont une synthèse, celle de Denis Monière; elle devrait normalement déboucher sur une histoire des classes sociales au Québec. 